\title{
On a non-local curve evolution problem in the plane
}

\author{
Lishang Jiang and Shengliang Pan
}

\begin{abstract}
This paper deals with a new curvature flow for closed convex plane curves which shortens the length of the evolving curve but expands the area it bounds and makes the evolving curve more and more circular during the evolution process. And the final shape of the evolving curve will be a circle (as the time $t$ goes to infinity). This flow is determined by a coupled system concerning both local and global geometric quantities of the evolving curve.
\end{abstract}

Mathematics Subject Classification: 35K15, 35K65, 53A04

\section{Introduction}

Traditionally, differential geometry has been the study of curved spaces or shapes in which, for the most part, time did not play a role. In the last few decades, on the other hand, geometers and geometric analysts have made great strides in understanding shapes that evolve in time; see, for instance, $[1-6,8,10,12-16,18,24,25]$, etc. Among them, perhaps, the simplest case (but already a very subtle one) is the curve-shortening flow in the plane studied by Gage and Hamilton [12] and Grayson [13]. The book by Kai-Seng Chou(Tso) and Xi-Ping Zhu [7] provides an excellent and unified account of many results related to flowing curves by curvature.

The aim of this paper is, motivated by $[11,17,19]$, to investigate a nonlocal curve evolution problem in the plane. Let $X(u, t):[a, b] \times[0, \infty) \rightarrow R^{2}$ be a family of closed planar curves with $X(u, 0)=X_{0}(u)$ being a closed convex curve. Consider the following evolution problem:

$$
\begin{aligned}
X_{t} & =\left(k-\frac{L}{2 A}\right) N, \\
L(t) & =\int_{a}^{b}\left|X_{u}\right| d u=\int_{a}^{b} \sqrt{\left(\frac{\partial x}{\partial u}\right)^{2}+\left(\frac{\partial y}{\partial u}\right)^{2}} d u, \\
A(t) & =\frac{1}{2} \int_{a}^{b}\left(x \frac{\partial y}{\partial u}-y \frac{\partial x}{\partial u}\right) d u, \\
X(u, 0) & =X_{0}(u),
\end{aligned}
$$


where $x=x(u, t)$ and $y=y(u, t)$ are coordinate components of the position vector $X(u, t), k$ is the signed curvature of the evolving curve, $L=L(t)$ and $A=A(t)$ are, respectively, the length of the curve and the area it bounds at time $t$ and $N$ the unit inward pointing normal vector along the curve. We will give our investigation along the ideas in [10-12], compared to which the geometric estimates are somewhat easier in the present case and the analytic estimates a bit harder.

This paper is organized as follows. Section 2 is devoted to prove that if the evolving convex curve does not develop singularities then it converges to a circle in the Hausdorff metric as $t$ goes to infinity (Theorem 2.8). Compared with the ideas in [10] and [12], our concepts are different in that the limiting curve in our case is a circle not a point and that $t$ does go to infinity not a finite time. In Section 3, we shall first prove in Theorem 3.3 that the evolution problem is equivalent to the following initial value problem of a coupled differentia-integral system:

$$
\begin{aligned}
k_{t} & =k^{2} k_{\theta \theta}+\left(k^{2}-\frac{L}{2 A} k\right) k, \\
L_{t} & =-\int_{0}^{2 \pi} k d \theta+\frac{\pi L}{A}, \\
A_{t} & =-2 \pi+\frac{L^{2}}{2 A} \\
k(\theta, 0) & =k_{0}(\theta)>0, \\
L(0) & =L_{0}=\int_{0}^{2 \pi} \frac{d \theta}{k_{0}(\theta)}>0, \\
A(0) & =A_{0}=-\int_{0}^{2 \pi}\left\langle X_{0}(\theta), N\right\rangle \frac{d \theta}{k_{0}(\theta)}>0,
\end{aligned}
$$

where

$$
X_{0}(\theta)=\left(\int_{0}^{\theta} \frac{\cos \varphi}{k_{0}(\varphi)} d \varphi, \int_{0}^{\theta} \frac{\sin \varphi}{k_{0}(\varphi)} d \varphi\right)
$$

and $N=(-\sin \theta, \cos \theta)$. And then, using maximum principal, Picard's theorem in ODEs and the Leray-Schauder fixed point theorem (for details, see [22]) yields that this initial value problem is locally solvable (Theorem 3.4).

In Section 4, just as Gage and Hamilton in [12] have done, we make the geometric, integral and pointwise estimates about the evolving convex curve and then use them to prove that all higher derivatives of curvature $k$ with respect to $\theta$ are uniformly bounded and therefore all higher derivatives of $k$ 
with respect to $t$ are also bounded. Thus we can conclude in Theorem 4.12 that the initial value problem (1.5)-(1.10) is also globally solvable.

In the last section, mimicking the proofs of Section 5 of [12] one can show that the evolving curve converges to a circle in the " $C$ " metric. Therefore, we get the following main result of the present paper.

Main theorem. A closed convex plane curve which evolves according to (1.1)-(1.4) remains convex, expands the enclosed area but shortens its length during the evolution process and converges to a circle in the $C^{\infty}$ metric as $t$ goes to infinity.

Remark 1.1. It is known that if a one-parameter family of closed convex plane curves $\gamma(\cdot, t)$ are evolved by the general flow $X_{t}=W \in \mathbf{R}^{2}$, then the length $L(t)$ and the enclosed area $A(t)$ of $\gamma(\cdot, t)$ will satisfy the following

$$
\frac{d L}{d t}=-\int_{\gamma(\cdot, t)}\langle W, k N\rangle d s
$$

and

$$
\frac{d A}{d t}=-\int_{\gamma(\cdot, t)}\langle W, N\rangle d s
$$

where $N$ is the unit inward normal and $k$ is the curvature with respect to $N$. In particular, the isoperimetric ratio $L^{2}(t) /(4 \pi A(t))$ will satisfy

$$
\frac{d}{d t}\left(\frac{L^{2}(t)}{4 \pi A(t)}\right)=-\frac{L(t)}{2 \pi A(t)} \int_{\gamma(\cdot, t)}\left\langle W,\left(k-\frac{L(t)}{2 A(t)}\right) N\right\rangle d s .
$$

Hence for the obvious choice $W=\left(k-\frac{L(t)}{2 A(t)}\right) N$, the isoperimetric ratio is decreasing under the present flow. This is to say that the closed convex curves are becoming more and more circular.

Remark 1.2. The present evolution problem has the following physical interpretations. Equation (1.1) represents the motion of a super elastic rubber band, with small mass in a viscous (high friction) medium, which surrounds an expanding material while forces the boundary to decrease.

Remark 1.3. In our case, however, we cannot obtain an analogue of Grayson's result now, i.e., it is not known for us whether a simple closed curve evolves smoothly to a convex one under (1.1)-(1.4).

Remark 1.4. It is also interesting to consider the similar evolution problem in higher dimensions. 


\section{Final shape of the evolving curve}

Let $X_{0}(u):[a, b] \longrightarrow R^{2}$ be a convex closed curve in the plane and $X(u, t)=$ $(x(u, t), y(u, t)):[a, b] \times[0, \infty) \longrightarrow R^{2}$ be a family of closed planar curves which evolves under (1.1)-(1.4).

Let $g(u, t)=\left|X_{u}\right|=\left(x_{u}^{2}+y_{u}^{2}\right)^{1 / 2}$ denote the metric along the curve, then the element of arc-length is given by $d s=g(u, t) d u$, or formally

$$
\frac{\partial}{\partial s}=\frac{1}{g} \frac{\partial}{\partial u}, \quad \frac{\partial s}{\partial u}=g .
$$

It is important that the partial derivative with respect to $t$ is taken keeping the parameter $u$ fixed. The operator $\frac{\partial}{\partial s}$ is not a partial derivative and this makes us change the parameters $u$ and $t$ to the new parameters $\theta$ and $\tau$ to simplify the equation for curvature in Lemma 2.2 below. The tangent $T$, normal $N$, orientation $\theta$, curvature $k$ and length $L$ of the curve and area $A$ it bounds are defined in the standard way:

$$
\begin{aligned}
T & =\frac{\partial X}{\partial s}=\frac{1}{g} \frac{\partial X}{\partial u}, \quad k=\frac{\partial \theta}{\partial s}=\frac{1}{g} \frac{\partial \theta}{\partial u}, \quad N=\frac{1}{k} \frac{\partial T}{\partial s}=\frac{1}{k g} \frac{\partial T}{\partial u}, \\
\theta & =\angle(T, x), \quad L(t)=\int_{a}^{b} g(u, t) d u=\oint d s, \\
A(t) & =\frac{1}{2} \oint x d y-y d x=-\frac{1}{2} \oint\langle X, N\rangle d s .
\end{aligned}
$$

According to $[7, \S 1.3]$ or $[21]$ one gets immediately

Lemma 2.1. The geometric quantities of the evolving curve evolve as

$$
\begin{aligned}
& \frac{\partial g}{\partial t}=-\left(k-\frac{L}{2 A}\right) k g, \quad \frac{\partial T}{\partial t}=\frac{\partial k}{\partial s} N, \quad \frac{\partial N}{\partial t}=-\frac{\partial k}{\partial s} T \\
& \frac{\partial \theta}{\partial t}=\frac{\partial k}{\partial s}=\frac{1}{g} \frac{\partial k}{\partial u}, \quad \frac{\partial k}{\partial t}=\frac{\partial^{2} k}{\partial s^{2}}+\left(k-\frac{L}{2 A}\right) k^{2} \\
& \frac{d L}{d t}=-\oint k^{2} d s+\frac{\pi L}{A}, \quad \frac{d A}{d t}=-2 \pi+\frac{L^{2}}{2 A} .
\end{aligned}
$$

Since $s=s(u, t)$ depends on both $u$ and $t$, the curvature evolution equation above is difficult to handle. Since the changing of the tangential components of the velocity vector $X_{t}$ affects only the parameterization, not the geometric shapes of the evolving curve (see, for example, $[7,11,12]$ or [23]), one may choose a suitable tangential component $\alpha=\alpha(u, t)$ so that the geometric analysis of the evolving curves can be simplified, i.e., we may consider 
the following evolution problem which is equivalent to (1.1)-(1.4):

$$
\begin{aligned}
& X_{t}=\alpha T+\left(k-\frac{L}{2 A}\right) N, \\
& L=\int_{a}^{b}\left|X_{u}\right| d u, \\
& A=\frac{1}{2} \oint x d y-y d x, \\
& X(u, 0)=X_{0}(u) .
\end{aligned}
$$

Similar to Lemma 2.1 one has

$$
\begin{aligned}
& \frac{\partial g}{\partial t}=\alpha_{u}-\left(k-\frac{L}{2 A}\right) k g, \\
& \frac{\partial T}{\partial t}=\left(\alpha k+\frac{\partial k}{\partial s}\right) N, \\
& \frac{\partial N}{\partial t}=-\left(\alpha k+\frac{\partial k}{\partial s}\right) T, \\
& \frac{\partial \theta}{\partial t}=\alpha k+\frac{\partial k}{\partial s}=\alpha k+\frac{1}{g} \frac{\partial k}{\partial u}, \\
& \frac{\partial k}{\partial t}=\frac{\partial^{2} k}{\partial s^{2}}+\alpha \frac{\partial k}{\partial s}+\left(k-\frac{L}{2 A}\right) k^{2}, \\
& \frac{d L}{d t}=-\oint k^{2} d s+\frac{\pi L}{A}=-\int_{0}^{2 \pi} k d \theta+\frac{\pi L}{A}, \\
& \frac{d A}{d t}=-2 \pi+\frac{L^{2}}{2 A} .
\end{aligned}
$$

Observing that the evolution equations for $L$ and $A$ are both independent of $\alpha$. Generally speaking, $\theta$ is a function of $u$ and $t$. In order to make $\theta$ independent of the time $t$, it can be seen from (2.8) that we should take the tangential component function $\alpha$ as

$$
\alpha=-\frac{1}{k} \frac{\partial k}{\partial s}=-\frac{\partial k}{\partial \theta}
$$

And then from (2.6) and (2.7), $T$ and $N$ are both independent of $t$, and the evolution equation of curvature can be expressed in terms of $\theta$ and $t$.

\section{Lemma 2.2.}

$$
\frac{\partial k}{\partial t}=k^{2} \frac{\partial^{2} k}{\partial \theta^{2}}+k^{3}-\frac{L}{2 A} k^{2}
$$


Proof. From (2.9), (2.12) and Lemma 2.1 we get

$$
\begin{aligned}
\frac{\partial k}{\partial t} & =\frac{\partial}{\partial s}\left(k \frac{\partial k}{\partial \theta}\right)-\frac{\partial k}{\partial \theta} \frac{\partial k}{\partial s}+\left(k-\frac{L}{2 A}\right) k^{2} \\
& =k \frac{\partial}{\partial s}\left(\frac{\partial k}{\partial \theta}\right)+\left(k-\frac{L}{2 A}\right) k^{2}=k^{2} \frac{\partial^{2} k}{\partial \theta^{2}}+\left(k-\frac{L}{2 A}\right) k^{2}
\end{aligned}
$$

From Theorem 2.4 below we can change the parameters $(u, t)$ into $(\theta, \tau)$ so that $\tau=t$. The point is that $\partial / \partial t \neq \partial / \partial \tau$, because $\partial / \partial t$ is the partial derivative with $u$ fixed, and $\partial / \partial \tau$ is the derivative with $\theta$ fixed. In what follows we will give our discussion in this new coordinate system and for simplicity, we replace $\tau$ by $t$. In $\theta$ and $t$ coordinates the evolution equations for curvature $k$, length $L$ and area $A$ are also given by (2.13), (2.10) and (2.11), respectively.

In the rest of this paper, we will focus our attention on the following evolution problem which is also equivalent to (1.1)-(1.4):

$$
\begin{aligned}
X_{t} & =-\frac{\partial k}{\partial \theta} T+\left(k-\frac{L}{2 A}\right) N, \\
L & =\int_{0}^{2 \pi}\left|X_{\theta}\right| d \theta \\
A & =\frac{1}{2} \int_{0}^{2 \pi}\left(x \frac{\partial y}{\partial \theta}-y \frac{\partial x}{\partial \theta}\right) d \theta, \\
X(\theta, 0) & =X_{0}(\theta)
\end{aligned}
$$

where $X(\theta, t)=(x(\theta, t), y(\theta, t))$ is the position vector of the curve.

This section is the first step which is devoted to proving that the evolving curve remain convex during the evolution process and the final shape is a circle in the Hausdorff metric if the initial curve is convex.

Lemma 2.3. If $X(\theta, t): S^{1} \times[0, \infty) \longrightarrow R^{2}$ evolves under $(2.14)-(2.17)$ then $\frac{L}{A}$ decreases during the evolution process.

Proof. Using the fact that $\int k d s=2 \pi$ and the Cauchy-Schwartz' inequality and the classical isoperimetric inequality, it follows from 
(2.10) and (2.11) that

$$
\begin{aligned}
\frac{d}{d t}\left(\frac{L}{A}\right) & =\frac{1}{A}\left(-\oint k^{2} d s+\frac{\pi L}{A}\right)-\frac{L}{A^{2}}\left(-2 \pi+\frac{L^{2}}{2 A}\right) \\
& \leq \frac{1}{A}\left(-\frac{4 \pi^{2}}{L}+\frac{\pi L}{A}\right)-\frac{L}{A^{2}}\left(-2 \pi+\frac{L^{2}}{2 A}\right) \\
& =\frac{\left(L^{2}-4 \pi A\right)\left(2 \pi A-L^{2}\right)}{2 A^{3} L} \leq 0,
\end{aligned}
$$

which gives us the desired result.

Theorem 2.4. A strictly convex curve evolving according to (2.14)-(2.17) remains convex during the evolution process.

Proof. We will use the maximum principle to show that the curvature remains positive for all time if the initial curvature is positive. Let $W(\theta, t)=$ $k(\theta, t) e^{\mu t}$, with $\mu$ a constant to be chosen later. Then $W$ satisfies the following equation:

$$
W_{t}=k^{2} W_{\theta \theta}+\left(k^{2}-\frac{L}{2 A} k+\mu\right) W .
$$

The coefficient of $W$ is a quadratic polynomial in $k$ whose discriminant is $\left(L^{2} / 4 A^{2}\right)-4 \mu$. From Lemma 2.3 we see that, for all $t>0, L / A \leq L_{0} / A_{0}$. If we choose $\mu>L_{0}^{2} / 16 A_{0}^{2}$, then the discriminant is negative and the coefficient of $W$ is positive. We will choose $\mu>L_{0}^{2} / 16 A_{0}^{2}$ so that this coefficient is always positive.

Now, let

$$
W_{\min }(t)=\inf \{W(\theta, t): 0 \leq \theta \leq 2 \pi\},
$$

and suppose that there exists $\eta$, where $0<\eta<W_{\min }(0)=k_{\min }(0)$, such that at some point $t>0, W_{\min }(t)=\eta$. Let $t_{0}=\inf \left\{t: W_{\min }(t)=\eta\right\}$, then, the continuity of $W$ assures that this minimum $\eta$ is achieved for the first time at $\left(\theta_{0}, t_{0}\right)$ and at this point

$$
W_{t} \leq 0, \quad W_{\theta \theta} \geq 0 \quad \text { and } \quad W=\eta>0,
$$

which contradicts with the fact that $W$ satisfies (2.18) and implies that, for all $t>0, W_{\min }(t) \geq W_{\min }(0)$. Hence, for all $t>0$, we have

$$
k(\theta, t) \geq k_{\min }(t)=W_{\min }(t) e^{-\mu t} \geq k_{\min }(0) e^{-\mu t}>0 .
$$

Thus completes the proof. 
Lemma 2.5. Under the evolution defined by (2.14)-(2.17) with the initial curve being convex, then the length of the evolving curve decreases and the area enclosed by the curve increases during the evolution process.

Proof. In [9], Gage has proved that for convex closed plane curves, there holds

$$
\int k^{2} d s \geq \frac{\pi L}{A}
$$

which is now called by us Gage's inequality. Theorem 2.4 guarantees that Gage's inequality holds for each curve during the evolution process, and thus from (2.10) and Gage's inequality it follows that

$$
L_{t}=-\int k^{2} d s+\frac{\pi L}{A} \leq 0,
$$

which proves the first assertion. As for the area $A$, using (2.11) and the classical isoperimetric inequality, one obtains $A_{t} \geq 0$, thus completes the proof.

This lemma tells us that $L \leq L_{0}$ and $A \geq A_{0}$, which together with the classical isoperimetric inequality gives us:

$$
4 \pi A_{0} \leq 4 \pi A \leq L^{2} \leq L_{0}^{2}
$$

and therefore

$$
\begin{aligned}
& \frac{4 \pi}{L_{0}} \leq \frac{4 \pi}{L} \leq \frac{L}{A} \leq \frac{L_{0}}{A_{0}}, \\
& 4 \pi \leq \frac{L^{2}}{A} \leq \frac{L_{0}^{2}}{A_{0}}
\end{aligned}
$$

where $L_{0}$ and $A_{0}$ are, respectively, the length of the initial curve and the area it encloses. Thus $L$ and $A$ are both monotonic and bounded functions, there exist $\bar{L}\left(\sqrt{4 \pi A_{0}} \leq \bar{L} \leq L_{0}\right)$ and $\bar{A}\left(A_{0} \leq \bar{A} \leq \frac{L_{0}^{2}}{4 \pi}\right)$ such that

$$
\lim _{t \rightarrow \infty} L(t)=\bar{L}, \quad \lim _{t \rightarrow \infty} A(t)=\bar{A}
$$

and therefore $\frac{L}{A}$ has a finite positive limit $\frac{\bar{L}}{A}$ as $t$ goes to infinity.

The following result is a direct corollary to Lemma 2.5 .

Corollary 2.6. If a convex curve evolves according to (2.14)-(2.17), then the isoperimetric deficit $L^{2}-4 \pi A$ is decreasing during the evolution process and converges to zero as $t$ goes to infinity. 
Proof. Evidently, from the proof of Lemma 2.5,

$$
\frac{d}{d t}\left(L^{2}-4 \pi A\right)=2 L L_{t}-4 \pi A_{t} \leq 0
$$

and thus $L^{2}-4 \pi A$ is decreasing. Now we employ Lemma 2.5 and (2.10), (2.11) and (2.20) to make further estimate:

$$
\begin{aligned}
\frac{d}{d t}\left(L^{2}-4 \pi A\right) & =2 L L_{t}-4 \pi A_{t} \leq-4 \pi \frac{L^{2}-4 \pi A}{2 A} \\
& \leq-\frac{8 \pi^{2}}{L_{0}^{2}}\left(L^{2}-4 \pi A\right)
\end{aligned}
$$

Integrating this yields

$$
L^{2}-4 \pi A \leq\left(L_{0}^{2}-4 \pi A_{0}\right) e^{-\left(8 \pi^{2}\right) /\left(L_{0}^{2}\right) t} .
$$

From the classical isoperimetric inequality and (2.23), we see that $L^{2}-4 \pi A$ converges to zero as $t$ goes to infinity.

This result and the isoperimetric inequality imply that the limiting curve is a circle. Furthermore, one can, in a similar way, prove the next corollary which shows that the isoperimetric ratio is decreasing and converges to $4 \pi$ as $t$ goes to infinity.

Corollary 2.7. Under the same hypothesis as in Corollary 2.6, the isoperimetric ratio $\frac{L^{2}}{A}$ is decreasing to $4 \pi$ as $t$ goes to infinity.

Employing Corollary 2.7 and the Bonnesen inequality (see [20]) yields the following theorem easily, we omit the details of the proof (which is similar to that of [11, Corollary 2.5]) here.

Theorem 2.8. If an evolving convex curve does not develop singularities then it converges to a circle in the Hausdorff metric.

From Theorem 2.8 one can conclude that there exists a positive number $R$ such that

$$
\lim _{t \rightarrow \infty} L(t)=2 \pi R, \quad \lim _{t \rightarrow \infty} A(t)=\pi R^{2} .
$$

Remark 2.9. From (2.23) it follows that if the initial curve is a circle then the evolving curve $X(u, t)$ is always a circle the same as the initial one for each time $t$. That is to say, the initial circle remains the same during the evolution process. 


\section{Short-time existence for convex curve}

In this section, we will first reduce the evolution problem for the curve to an initial value problem of certain nonlinear differential equations and then consider the short time existence and uniqueness for the later initial value problem.

First, we prove a result which can be considered as a refinement of Theorem 2.4.

Theorem 3.1. Let

$$
k_{\min }(t)=\inf \{k(\theta, t): 0 \leq \theta \leq 2 \pi\},
$$

then the function $k_{\min }(t) e^{\left(L^{2}(t)\right) /\left(16 A^{2}(t)\right) t}$ is increasing during the evolution process.

Proof. Set $W(\theta, t)=k(\theta, t) e^{\mu t}$, where $\mu$ is a constant to be determined later, then $W$ satisfies the following problem:

$$
\begin{aligned}
W_{t} & =k^{2} W_{\theta \theta}+\left(k^{2}-\frac{L}{2 A} k+\mu\right) W, \\
\left.W\right|_{t=0} & =k_{0}(\theta)>0 .
\end{aligned}
$$

Let $G(k, \mu)=k^{2}-\frac{L}{2 A} k+\mu$, then from Lemma 3.3

$$
G(k, \mu)>\left(k-\frac{L}{4 A}\right)^{2}+\mu-\frac{L_{0}^{2}}{16 A_{0}^{2}},
$$

taking $\mu=L_{0}^{2} / 16 A_{0}^{2}$ so that $G(k, \mu)>0$.

Now, let

$$
W_{\min }(t)=\inf \{W(\theta, t): 0 \leq \theta \leq 2 \pi\}
$$

(a) First, similar to the proof of Theorem 2.4, we have $W_{\min }(t) \geq W_{\min }(0)$ for all $t>0$.

(b) Next, we show that $W_{\min }(t)>W_{\min }(0)$ for all $t>0$.

The proof is also by contradiction. If not the case, there exists a point $t^{*}>0$ such that $W_{\min }\left(t^{*}\right)=W_{\min }(0)$. We apply the maximum principle to the set $S^{1} \times\left[0, t^{*}\right]$. Similar to the proof of Theorem 2.4, we can arrive at a contradiction observing that at the point $\left(\theta^{*}, t^{*}\right), W(\theta, t)$ assume its minimum.

(c) Now, we show that $W_{\min }(t)$ increases strictly. 
The proof is again by contradiction. If not, there exists a time interval $\left(t_{1}, t_{2}\right), 0 \leq t_{1}<t_{2}$ such that $W_{\min }\left(t_{1}\right) \geq W_{\min }\left(t_{2}\right)$. We apply the maximum principle to the set $S^{1} \times\left[t_{1}, t_{2}\right]$, from (a) and (b), substituting $t=t_{1}$ for $t=0$ and $t=t_{2}$ for $t=t^{*}$, we have $W_{\min }\left(t_{2}\right)>W_{\min }\left(t_{1}\right)$, a contradiction.

(d) Finally, we show further that $k_{\min }(t) e^{\left(L^{2}(t)\right) /\left(16 A^{2}(t)\right) t}$ is a increasing function of $t$.

Dividing the time interval $[0, t]$ into $N$ parts, $t=N \Delta t$ :

$$
\Delta: 0=t_{0}<t_{1}=\Delta t<t_{2}<\cdots<t_{N}=t .
$$

Considering the following problem on the set $S^{1} \times\left[t_{l}, t_{l+1}\right]$ :

$$
\begin{aligned}
k_{t} & =k^{2} k_{\theta \theta}+\left(k^{2}-\frac{L}{2 A} k\right) k, \\
\left.k\right|_{t=t_{l}} & =k\left(\theta, t_{l}\right)>0 .
\end{aligned}
$$

where $l=0,1, \ldots, N-1$. Let $W_{l}(\theta, t)=k(\theta, t) e^{\mu t+\left(\mu_{l-1}-\mu\right) t_{l}}$, where $\mu_{l-1}$ is determined by induction, $\mu_{-1}=0, \quad \mu_{0}=L_{0}^{2} / 16 A_{0}^{2}$. Then, $W_{l}$ $(\theta, t)$ will satisfy

$$
\begin{aligned}
\left(W_{l}\right)_{t} & =k^{2}\left(W_{l}\right)_{\theta \theta}+\left(k^{2}-\frac{L}{2 A} k+\mu\right) W_{l}, \\
\left.W_{l}\right|_{t=t_{l}} & =k\left(\theta, t_{l}\right) e^{\mu_{l-1} t_{l}}>0 .
\end{aligned}
$$

Taking

$$
\mu=\mu_{l}=\frac{L^{2}\left(t_{l}\right)}{16 A^{2}\left(t_{l}\right)}>\frac{L^{2}(t)}{16 A^{2}(t)},
$$

for $t_{l}<t \leq t_{l+1}$, then from (a), (b) and (c) we obtain that the function

$$
\left(W_{l}\right)_{\min }(t)=\inf \left\{W_{l}(\theta, t): 0 \leq \theta \leq 2 \pi\right\}
$$

is increasing strictly for $t_{l} \leq t \leq t_{l+1}$.

Define $W_{\Delta}(t)$ :

$$
W_{\Delta}(t)=\left(W_{l}\right)_{\min }(t)=k_{\min }(t) e^{\mu_{l}\left(t-t_{l}\right)+\mu_{l-1} t_{l}},
$$

where $t_{l} \leq t \leq t_{l+1}, 0 \leq l \leq N-1$, therefore $W_{\Delta}(t)$ is increasing strictly. Then

$$
\lim _{\Delta \rightarrow 0} W_{\Delta}(t)=k_{\min }(t) e^{\left(L^{2}(t)\right) /\left(16 A^{2}(t)\right) t}
$$

is increasing. 
From this theorem, $k_{\min }(t) e^{\left(L^{2}(t)\right) /\left(16 A^{2}(t)\right) t} \geq k_{\min }(0)$ for all $t>0$, and therefore for all $(\theta, t) \in S^{1} \times[0, \infty)$ we have

$$
k(\theta, t) \geq k_{\min }(t) \geq k_{\min }(0) e^{-\left(L^{2}(t)\right) /\left(16 A^{2}(t)\right) t}>0
$$

Now we check the "closing condition" for each curve in the evolving process.

Lemma 3.2. If $k_{0}(\theta)=k(\theta, 0)>0$ satisfies

$$
\int_{0}^{2 \pi} \frac{e^{i \theta}}{k_{0}(\theta)} d \theta=0
$$

then, for each $t$, the solution $k(\theta, t)$ to (2.13) (i.e., (3.3) below) satisfies

$$
\int_{0}^{2 \pi} \frac{e^{i \theta}}{k(\theta, t)} d \theta=0
$$

Proof. From Theorem 3.1 we can rewrite (2.13) as

$$
\left(-\frac{1}{k}\right)_{t}=k_{\theta \theta}+k-\frac{L}{2 A}
$$

Integrating this over the set $[0,2 \pi] \times[0, t]\left(\right.$ times $\left.e^{i \theta}\right)$ yields

$$
\int_{0}^{2 \pi}\left(-\frac{e^{i \theta}}{k(\theta, t)}\right) d \theta-\int_{0}^{2 \pi}\left(-\frac{e^{i \theta}}{k_{0}(\theta)}\right) d \theta=\int_{0}^{t} \int_{0}^{2 \pi} e^{i \theta}\left(k_{\theta \theta}+k-\frac{L}{2 A}\right) d \theta=0
$$

where we have done the integration by parts in the last equation.

Hence, we can reduce the evolution problem for the curve to an initial value problem of some nonlinear differentio-integral equations in the following theorem.

Theorem 3.3. The evolution problem (2.14)-(2.17) for the convex curves is equivalent to the following initial value problem.

Find $k=k(\theta, t): S^{1} \times[0, \infty) \longrightarrow R^{+}, \quad L=L(t):[0, \infty) \longrightarrow R^{+}$and $A=A(t):[0, \infty) \longrightarrow R^{+}$such that

(i) $k \in C^{2}+\sigma, 1+\sigma / 2\left(S^{1} \times\left[0, T^{*}\right)\right), L \in C^{1}\left(\left[0, T^{*}\right)\right)$ and $A \in C^{1}\left(\left[0, T^{*}\right)\right)$ for all $T^{*}>0$. 
(ii)

$$
\begin{aligned}
\frac{\partial k}{\partial t} & =k^{2} \frac{\partial^{2} k}{\partial \theta^{2}}+k^{3}-\frac{L}{2 A} k^{2}, \\
\frac{d L}{d t} & =-\int_{0}^{2 \pi} k d \theta+\frac{\pi L}{A}, \\
\frac{d A}{d t} & =-2 \pi+\frac{L^{2}}{2 A}
\end{aligned}
$$

(iii) (a) $k(\theta, 0)=k_{0}(\theta) \in C^{1+\sigma}\left(S^{1}\right), k_{0}(\theta)>0$ and $\int_{0}^{2 \pi} e^{i \theta} / k_{0}(\theta) d \theta=0$; and (b) $L(0)=L_{0}=\int_{0}^{2 \pi} \frac{d \theta}{k_{0}(\theta)}>0$, and $A(0)=A_{0}>0\left(A_{0}\right.$ is defined in (3.9) below).

Proof. Equations (2.10), (2.11) and (2.13) tells us that given a solution to the evolution problem (2.14)-(2.17), the curvature, length and area functions of the evolving curves, expressed in $\theta$ and $t$ coordinates, will satisfy (ii) and (iii).

Conversely, given a solution $k(\theta, t), L(t)$ and $A(t)$ to the initial value problem of the differentio-integral system, for each $t \geq 0$ the associated curve $X(\theta, t)=(x(\theta, t), y(\theta, t))$, up to translation, defined by

$$
x(\theta, t)=\int_{0}^{\theta} \frac{\cos \phi}{k(\phi, t)} d \phi, \quad y(\theta, t)=\int_{0}^{\theta} \frac{\sin \phi}{k(\phi, t)} d \phi
$$

satisfies the evolution equation

$$
\frac{\partial X}{\partial t}=-\frac{\partial k}{\partial \theta} T+\left(k-\frac{L}{2 A}\right) N
$$

Now, a direct calculation implies that the curvature of the associated curve $X(\theta, t)$ is exactly $k(\theta, t)$.

Next, we check that the length $\tilde{L}(t)$ of the associated curve is $L(t)$. In fact, from (3.6) it follows that

$$
\tilde{L}(t)=\int_{0}^{2 \pi} \sqrt{x_{\theta}^{2}+y_{\theta}^{2}} d \theta=\int_{0}^{2 \pi} \sqrt{\frac{\cos ^{2} \theta}{k^{2}}+\frac{\sin ^{2} \theta}{k^{2}}} d \theta=\int_{0}^{2 \pi} \frac{d \theta}{k} .
$$

Using curvature evolution equation (3.3) we get

$$
\frac{1}{k}=\frac{1}{k_{0}}-\int_{0}^{t}\left(k_{\theta \theta}+k-\frac{L}{2 A}\right) d \theta
$$


Taking (3.8) into (3.7) and exchanging the order of integration gives the desired result as follows

$$
\begin{aligned}
\tilde{L}(t) & =\int_{0}^{2 \pi}\left[\frac{1}{k_{0}(\theta)}-\int_{0}^{t}\left(k_{\theta \theta}+k-\frac{L}{2 A}\right) d t\right] d \theta \\
& =\int_{0}^{2 \pi} \frac{d \theta}{k_{0}(\theta)}-\int_{0}^{t} \int_{0}^{2 \pi} k_{\theta \theta} d \theta d t-\int_{0}^{t} \int_{0}^{2 \pi} k d \theta d t+\int_{0}^{t} \frac{\pi L}{A} d t \\
& =L_{0}+\int_{0}^{t}\left(-\int_{0}^{2 \pi} k d \theta+\frac{\pi L}{A}\right) d t=L_{0}+\int_{0}^{t} L_{t} d t=L(t)
\end{aligned}
$$

where we have used the length evolution equation (3.4) in the last but one equality.

Finally, we check that the area $\tilde{A}(t)$ bounded by the associated curve is also $A(t)$. In fact, by definition,

$$
\begin{aligned}
& \tilde{A}(t)=-\frac{1}{2} \oint\langle X, N\rangle d s=-\frac{1}{2} \int_{0}^{2 \pi}\langle X, N\rangle \frac{d \theta}{k}, \\
& \tilde{A}(0)=-\frac{1}{2} \int_{0}^{2 \pi}\left\langle X_{0}, N_{0}\right\rangle \frac{d \theta}{k_{0}(\theta)}=A_{0} .
\end{aligned}
$$

Therefore, we need only to show that $A_{t}=\tilde{A}_{t}$. From (3.8), (3.3) and the facts that

$$
N_{t}=0, \quad\langle T, N\rangle=0, \quad \int_{0}^{2 \pi} \frac{d \theta}{k}=\oint d s=L,
$$

and $L=-\int_{0}^{2 \pi}\langle X, N\rangle d \theta$, it follows that

$$
\begin{aligned}
\tilde{A}_{t}= & -\frac{1}{2} \int_{0}^{2 \pi}\left(\left\langle X_{t}, N\right\rangle+\left\langle X, N_{t}\right\rangle\right) \frac{d \theta}{k}+\frac{1}{2} \int_{0}^{2 \pi}\langle X, N\rangle \frac{k_{t}}{k^{2}} d \theta \\
= & -\frac{1}{2} \int_{0}^{2 \pi}\left(k-\frac{L}{2 A}\right) \frac{d \theta}{k}+\frac{1}{2} \int_{0}^{2 \pi}\langle X, N\rangle\left(k_{\theta \theta}+k-\frac{L}{2 A}\right) d \theta \\
= & \frac{1}{2}\left(-2 \pi+\frac{L^{2}}{2 A}\right)+\frac{1}{2} \int_{0}^{2 \pi}\langle X, N\rangle k_{\theta \theta} d \theta \\
& +\frac{1}{2} \int_{0}^{2 \pi}\langle X, N\rangle k d \theta+\frac{1}{2} \frac{L^{2}}{2 A}
\end{aligned}
$$


Integrating by parts and using the facts that $X_{\theta}=\frac{1}{k} T, N_{\theta}=-T, T_{\theta}=N$ gives us

$$
\begin{aligned}
\int_{0}^{2 \pi} & \langle X, N\rangle k_{\theta \theta} d \theta=\left.\langle X, N\rangle k_{\theta}\right|_{0} ^{2 \pi}-\int_{0}^{2 \pi}\left(\left\langle X_{\theta}, N\right\rangle+\left\langle X, N_{\theta}\right\rangle\right) k_{\theta} d \theta \\
\quad & \left.=\int_{0}^{2 \pi}\langle X, T\rangle k_{\theta} d \theta=\left.\langle X, T\rangle k\right|_{0} ^{2 \pi}-\int_{0}^{2 \pi}\left(\left\langle X_{\theta}, T\right\rangle+\left\langle X, T_{\theta}\right\rangle\right)\right) k d \theta \\
& =-2 \pi-\int_{0}^{2 \pi}\langle X, N\rangle k d \theta
\end{aligned}
$$

Taking this into (3.10) yields

$$
\tilde{A}_{t}=\frac{1}{2}\left(-2 \pi+\frac{L^{2}}{2 A}\right)+\frac{1}{2}\left(-2 \pi+\frac{L^{2}}{2 A}\right)=A_{t} .
$$

In [22], we have proved that the above initial value problem is locally solvable. (Comparing with the method used by Gage and Hamilton, we have applied the maximum principal, the Picard theorem in ODEs and the LeraySchauder fixed point theorem instead of the Nash-Moser inverse function technique).

Theorem 3.4. Suppose that $k(\theta, 0)=k_{0}(\theta) \in C^{2+\sigma}\left(S^{1}\right)$ and there exist positive constants $\alpha$ and $\beta$ such that $\alpha \leq k_{0}(\theta) \leq \beta$. Then there exists $T_{0}>0$ such that there are unique solutions $k \in C^{2}+\sigma, 1+\sigma / 2\left(Q_{0}\right), L \in C^{1}\left(\left[0, T_{0}\right]\right)$ and $A \in C^{1}\left(\left[0, T_{0}\right]\right)$ to the following initial value problem:

$$
\begin{aligned}
k_{t} & =k^{2} k_{\theta \theta}+\left(k^{2}-\frac{L}{2 A} k\right) k, \\
L_{t} & =-\int_{0}^{2 \pi} k(\theta, t) d \theta+\frac{\pi L}{A}, \\
A_{t} & =-2 \pi+\frac{L^{2}}{2 A} \\
k(\theta, 0) & =k_{0}(\theta), \\
L(0) & =L_{0}=\int_{0}^{2 \pi} \frac{d \theta}{k_{0}(\theta)}>0, \\
A(0) & =A_{0}=-\frac{1}{2} \int_{0}^{2 \pi}\left\langle X_{0}, N\right\rangle \frac{d \theta}{k_{0}(\theta)}>0,
\end{aligned}
$$

where $X_{0}=\left(\int_{0}^{\theta}(\cos \varphi) /\left(k_{0}(\varphi)\right) d \varphi, \int_{0}^{\theta}(\sin \varphi) /\left(k_{0}(\varphi)\right) d \varphi\right), N=(-\sin \theta, \cos \theta)$ and $Q_{0}=S^{1} \times\left[0, T_{0}\right], L_{0}$ and $A_{0}$ satisfy $L_{0}^{2}-4 \pi A_{0} \geq 0$. 


\section{Long-term existence for convex curve}

In this section, we will employ the standard method in curvature flow theory given in $[11,12,16,17]$ to derive uniform bound for the maximum curvature. Define the median curvature $k^{*}$ as

$$
k^{*}(t)=\sup \{\beta: k(\theta, t)>\beta \text { on some interval of length } \pi\} .
$$

From [12, Proposition 4.3.2], one has the geometric estimate. If the convex closed planar curve encloses an area $A$ and has length $L$ then $k^{*}(t)<\frac{L}{A}$. Observing that, in our case, the length $L=L(t)$ is decreasing and the area $A=A(t)$ increasing, the median curvature $k^{*}(t)$ is uniformly bounded in terms of the initial conditions.

Proposition 4.1 (Geometric estimate). In our case, $k^{*}(t)$ is uniformly bounded, $k^{*}(t)<\frac{L_{0}}{A_{0}}$.

Proposition 4.2 (Integral estimate). Since $k^{*}(t)$ is bounded on the time interval $\left[0, T^{*}\right)$, we have $\int_{0}^{2 \pi} \ln k(\theta, t) d \theta$ is bounded on $\left[0, T^{*}\right)$.

Proof. On one hand, using Theorem 3.1, for all $t>0$, we have

$$
\begin{aligned}
\int_{0}^{2 \pi} \ln k(\theta, t) d \theta & \geq \int_{0}^{2 \pi} \ln k_{\min }(t) d \theta=2 \pi \ln k_{\min }(t) \\
& \geq 2 \pi \ln \left[k_{\min }(0) e^{-L^{2} / 16 A^{2} t}\right] \geq 2 \pi \ln k_{\min }(0)-\frac{\pi}{8} \frac{L_{0}^{2}}{A_{0}^{2}} t
\end{aligned}
$$

On the other hand, in terms of the evolution equation for curvature and integrating by parts we get

$$
\begin{aligned}
\frac{d}{d t} \int_{0}^{2 \pi} \ln k(\theta, t) d \theta & =\int_{0}^{2 \pi}\left(k k_{\theta \theta}+k^{2}-\frac{L}{2 A} k\right) d \theta \\
& =\int_{0}^{2 \pi}\left(-k_{\theta}^{2}+k^{2}-\frac{L}{2 A} k\right) d \theta \leq \int_{0}^{2 \pi}\left(-k_{\theta}^{2}+k^{2}\right) d \theta
\end{aligned}
$$

The open set $U=\left\{\theta: k(\theta, t)>k^{*}(t)\right\}$ can be written uniquely as the union of a countable number of disjoint open intervals $I_{i}$, each of which 
must have length $\leq \pi$ by definition (4.1). On each interval $I_{i}$ we have

$$
\begin{aligned}
\int_{\bar{I}_{i}}\left(-k_{\theta}^{2}+k^{2}\right) d \theta & =\int_{\bar{I}_{i}}\left\{-\left[\left(k-k^{*}\right)_{\theta}\right]^{2}+k^{2}\right\} d \theta \\
& \left.\leq \int_{\bar{I}_{i}}\left[-(k-3) \quad k^{*}\right)^{2}+k^{2}\right] d \theta=\int_{\bar{I}_{i}}\left[2 k k^{*}-\left(k^{*}\right)^{2}\right] d \theta \leq 2 k^{*} \int_{\bar{I}_{i}} k d \theta,
\end{aligned}
$$

where we have used the Wirtinger inequality.

On the complement of $U, U^{c}$, we have the estimate $k \leq k^{*}$ and

$$
\int_{U^{c}}\left(-k_{\theta}^{2}+k^{2}\right) d \theta \leq k^{*} \int_{U^{c}} k d \theta
$$

Therefore, combining (4.2), (4.3) and (4.4) and using Proposition 4.1 we obtain

$$
\frac{d}{d t} \int_{0}^{2 \pi} \ln k(\theta, t) d \theta \leq 2 k^{*} \int_{0}^{2 \pi} k d \theta \leq \frac{2 L_{0}}{A_{0}} \int_{0}^{2 \pi} k d \theta .
$$

Now, using (3.4) and (2.21) yields

$$
\frac{d}{d t} \int_{0}^{2 \pi} \ln k(\theta, t) d \theta \leq \frac{2 L_{0}}{A_{0}}\left(\frac{\pi L}{A}-L_{t}\right) \leq \frac{2 \pi L_{0}^{2}}{A_{0}^{2}}-\frac{2 L_{0}}{A_{0}} L_{t}
$$

Finally, integrating this gives us

$$
\int_{0}^{2 \pi} \ln k(\theta, t) d \theta \leq \int_{0}^{2 \pi} \ln k_{0}(\theta) d \theta+\frac{2 \pi L_{0}^{2}}{A_{0}^{2}} t-\frac{2 L_{0}}{A_{0}}\left(L(t)-L_{0}\right)
$$

which completes the proof, noting that $\sqrt{4 \pi A_{0}} \leq L \leq L_{0}$.

Lemma 4.3. If $\int_{0}^{2 \pi} \ln k(\theta, t) d \theta$ is bounded on $\left[0, T^{*}\right)$, then for any $\delta>0$ we can find a constant $M$ such that $k(\theta, t) \leq M$ except on $\theta$ intervals of length $\leq \delta$. 
Proof. The proof is by contradiction. Suppose that, for any $M>0$, there exists an interval $[a, b]$ with $b-a>\delta$ such that on this interval $k>M$, then

$$
\begin{aligned}
\int_{0}^{2 \pi} \ln k(\theta, t) d \theta & =\int_{a}^{b} \ln k(\theta, t) d \theta+\int_{[0,2 \pi] \backslash[a, b]} \ln k(\theta, t) d \theta \\
& >(b-a) \ln M+(2 \pi-(b-a)) \ln k_{\min }(t) \\
& =2 \pi \ln k_{\min }(t)+(b-a)\left(\ln M-\ln k_{\min }(t)\right) \\
& >2 \pi \ln k_{\min }(t)+\delta\left(\ln M-\ln k_{\min }(t)\right) \\
& =\delta \ln M+(2 \pi-\delta) \ln k_{\min }(t) \\
& \geq \delta \ln M+(2 \pi-\delta) \ln k_{\min }(0)-(2 \pi-\delta) \frac{L_{0}^{2}}{16 A_{0}^{2}} t
\end{aligned}
$$

This gives us a contradiction when $M$ is very large.

Now, we prove the following "reverse Poincare inequality."

Lemma 4.4. We can find a constant $D$ such that

$$
\int_{0}^{2 \pi}\left(k_{\theta}\right)^{2} d \theta \leq \int_{0}^{2 \pi} k^{2} d \theta+D
$$

holds for $0 \leq t<T^{*}$.

Proof. We can calculate:

$$
\frac{d}{d t} \int_{0}^{2 \pi}\left[k^{2}-\left(k_{\theta}\right)^{2}\right] d \theta=2 \int_{0}^{2 \pi}\left(k k_{t}-k_{\theta} k_{\theta t}\right) d \theta=2 \int_{0}^{2 \pi}\left(k+k_{\theta \theta}\right) k_{t} d \theta
$$

From the evolution equation (3.3) for the curvature it follows that $k_{\theta \theta}+k=$ $\frac{L}{2 A}+\frac{k_{t}}{k^{2}}$. Taking this into (4.5) yields

$$
\begin{aligned}
\frac{d}{d t} \int_{0}^{2 \pi}\left(k^{2}-k_{\theta}^{2}\right) d \theta & =2 \int_{0}^{2 \pi}\left(\frac{L}{2 A}+\frac{k_{t}}{k^{2}}\right) k_{t} d \theta \\
& =\frac{L}{A} \int_{0}^{2 \pi} k_{t} d \theta+2 \int_{0}^{2 \pi} \frac{k_{t}^{2}}{k^{2}} d \theta \geq \frac{L}{A} \int_{0}^{2 \pi} k_{t} d \theta=\frac{L}{A} \frac{d}{d t} \int_{0}^{2 \pi} k d \theta \\
& =\left(\frac{L}{A} \int_{0}^{2 \pi} k d \theta\right)_{t}-\left(\frac{L}{A}\right)_{t} \int_{0}^{2 \pi} k d \theta \geq\left(\frac{L}{A} \int_{0}^{2 \pi} k d \theta\right)_{t},
\end{aligned}
$$


where we have used Lemma 2.3, therefore, by integration we have

$$
\begin{aligned}
& \int_{0}^{2 \pi}\left(k^{2}-k_{\theta}^{2}\right) d \theta-\int_{0}^{2 \pi}\left[k_{0}^{2}(\theta)-k_{\theta}^{2}(\theta, 0)\right] d \theta \\
\geq & \frac{L}{A} \int_{0}^{2 \pi} k d \theta-\frac{L_{0}}{A_{0}} \int_{0}^{2 \pi} k_{0} d \theta \geq-\frac{L_{0}}{A_{0}} \int_{0}^{2 \pi} k_{0} d \theta .
\end{aligned}
$$

and therefore

$$
\int_{0}^{2 \pi} k_{\theta}^{2} d \theta \leq \int_{0}^{2 \pi} k^{2} d \theta+\int_{0}^{2 \pi}\left[k_{\theta}^{2}(\theta, 0)+\frac{L_{0}}{A_{0}} k_{0}(\theta)-k_{0}^{2}(\theta)\right] d \theta .
$$

Letting

$$
D=\max \left\{0, \int_{0}^{2 \pi}\left[k_{\theta}^{2}(\theta, 0)+\frac{L_{0}}{A_{0}} k_{0}(\theta)-k_{0}^{2}(\theta)\right] d \theta\right\}
$$

yields the desired result.

Proposition 4.5 (Pointwise estimate). If $\int_{0}^{2 \pi} \ln k(\theta, t) d \theta$ is bounded on $\left[0, T^{*}\right)$, then $k(\theta, t)$ is uniformly bounded on $S^{1} \times\left[0, T^{*}\right)$.

Proof. Since $\int_{0}^{2 \pi} \ln k(\theta, t) d \theta$ is bounded on $\left[0, T^{*}\right)$, from Lemma 4.3 we have $k \leq M$ except on intervals length less than $\delta$. On such an interval

$$
\begin{aligned}
k(\phi) & =k(a)+\int_{a}^{\phi} \frac{\partial k}{\partial \theta} d \theta \leq k(a)+\int_{a}^{\phi}\left|\frac{\partial k}{\partial \theta}\right| d \theta \\
& \leq M+\sqrt{\delta}\left(\int k_{\theta}^{2} d \theta\right)^{1 / 2} \leq M+\sqrt{\delta}\left(\int k^{2} d \theta+D\right)^{1 / 2} .
\end{aligned}
$$

This shows that if $k_{\max }$ is the maximum of $k$, then

$$
k_{\max } \leq M+\sqrt{\delta}\left(2 \pi k_{\max }^{2}+D\right)^{1 / 2} \leq M+\sqrt{2 \pi \delta} k_{\max }+\sqrt{\delta D} .
$$

Choosing $\delta$ small we have $k_{\max } \leq 2 M$.

Therefore we have

Theorem 4.6. If $k: S^{1} \times\left[0, T^{*}\right) \longrightarrow R$ satisfies equation (3.3), then the curvature $k$ is uniformly bounded on $S^{1} \times\left[0, T^{*}\right)$.

Now, using estimates for the $L_{2}$ norms of the derivatives of $k$ with respect to $\theta$ similar to those in $[12, \S 4.4]$ we shall prove that all the higher derivatives 
of $k$ with respect to $\theta$ are also bounded under the assumption that $k$ is bounded on the time interval $\left[0, T^{*}\right)$. To simplify notation, we use "I" to denote the partial derivative with respect to $\theta$ in what follows, for example,

$$
k^{\prime}=\frac{\partial k}{\partial \theta}, \quad k^{\prime \prime}=\frac{\partial^{2} k}{\partial \theta^{2}}, \cdots
$$

Lemma 4.7. If $k$ is uniformly bounded on $\left[0, T^{*}\right)$, then $k^{\prime}=\frac{\partial k}{\partial \theta}$ is also bounded on the same interval.

Proof. Using the evolution equation (3.3) we calculate:

$$
\begin{aligned}
\frac{\partial^{2} k}{\partial t \partial \theta} & =\left(\frac{\partial k}{\partial t}\right)^{\prime}=\left(k^{2} k^{\prime \prime}+k^{3}-\frac{L}{2 A} k^{2}\right)^{\prime} \\
& =2 k k^{\prime} k^{\prime \prime}+k^{2} k^{\prime \prime \prime}+3 k^{2} k^{\prime}-\frac{L}{A} k k^{\prime} \\
& =k^{2} k^{\prime \prime \prime}+2 k k^{\prime} k^{\prime \prime}+\left(3 k^{2}-\frac{L}{A} k\right) k^{\prime}
\end{aligned}
$$

This implies that $k^{\prime}$ grows at most exponentially as can be seen by considering the PDE. satisfied by $e^{\mu t} k^{\prime}$ and choosing $\mu$ so that the maximum principle can be used. On a finite time interval $k^{\prime}$ remains bounded.

Lemma 4.8. If $k$ and $k^{\prime}$ are both bounded on the interval $\left[0, T^{*}\right)$, then so is $\int_{0}^{2 \pi}\left(k^{\prime \prime}\right)^{4} d \theta$.

Proof. Using the evolution equation and integrating by parts we get

$$
\begin{aligned}
\frac{d}{d t} \int_{0}^{2 \pi}\left(k^{\prime \prime}\right)^{4} d \theta= & 4 \int_{0}^{2 \pi}\left(k^{\prime \prime}\right)^{3} \frac{\partial k^{\prime \prime}}{\partial \partial t} d \theta=4 \int_{0}^{2 \pi}\left(k^{\prime \prime}\right)^{3}\left(\frac{\partial k}{\partial t}\right)^{\prime \prime} d \theta \\
= & 4 \int_{0}^{2 \pi}\left(k^{\prime \prime}\right)^{3}\left(k^{2} k^{\prime \prime}+k^{3}-\frac{L}{2 A} k^{2}\right)^{\prime \prime} d \theta \\
= & -12 \int_{0}^{2 \pi}\left(k^{2} k^{\prime \prime}+k^{3}-\frac{L}{2 A} k^{2}\right)^{\prime}\left(k^{\prime \prime}\right)^{2} k^{\prime \prime \prime} d \theta \\
= & -12 \int_{0}^{2 \pi}\left(k^{\prime \prime}\right)^{2} k^{\prime \prime \prime}\left(k^{2} k^{\prime \prime \prime}+2 k k^{\prime} k^{\prime \prime}+3 k^{2} k^{\prime}-\frac{L}{A} k k^{\prime}\right) d \theta \\
= & 12 \int_{0}^{2 \pi}\left[-k^{2}\left(k^{\prime \prime}\right)^{2}\left(k^{\prime \prime \prime}\right)^{2}-2 k k^{\prime}\left(k^{\prime \prime}\right)^{3} k^{\prime \prime \prime}\right. \\
& \left.-3 k^{2} k^{\prime}\left(k^{\prime \prime}\right)^{2} k^{\prime \prime \prime}+\frac{L}{A} k k^{\prime}\left(k^{\prime \prime}\right)^{2} k^{\prime \prime \prime}\right] d \theta
\end{aligned}
$$


Using the Peter-Paul inequality: for any $\varepsilon>0$

$$
a b \leq \varepsilon a^{2}+\frac{b^{2}}{4 \varepsilon}
$$

to bound the second, third and fourth terms by the first term and some additional penalty terms and observing that $\frac{L}{A} \leq \frac{L_{0}}{A_{0}}$ we obtain

$$
\frac{d}{d t} \int_{0}^{2 \pi}\left(k^{\prime \prime}\right)^{4} \leq C_{1} \int_{0}^{2 \pi}\left(k^{\prime}\right)^{2}\left(k^{\prime \prime}\right)^{4}+C_{2} \int_{0}^{2 \pi} k^{2}\left(k^{\prime}\right)^{2}\left(k^{\prime \prime}\right)^{2}+C_{3} \int_{0}^{2 \pi}\left(k^{\prime}\right)^{2}\left(k^{\prime \prime}\right)^{2} .
$$

Using the uniform bounds on $k$ and $k^{\prime}$ and the fact that

$$
\int_{0}^{2 \pi}\left(k^{\prime \prime}\right)^{2} \leq \sqrt{2 \pi}\left[\int_{0}^{2 \pi}\left(k^{\prime \prime}\right)^{4}\right]^{1 / 2}
$$

we see that $\int_{0}^{2 \pi}\left(k^{\prime \prime}\right)^{4}$ grows at most exponentially and therefore remains finite on a finite interval.

Lemma 4.9. If $k, k^{\prime}$ and $\int_{0}^{2 \pi}\left(k^{\prime \prime}\right)^{4}$ are bound, so is $\int_{0}^{2 \pi}\left(k^{\prime \prime \prime}\right)^{2}$.

Proof. Similarly, we calculate:

$$
\begin{aligned}
\frac{d}{d t} \int\left(k^{\prime \prime \prime}\right)^{2}= & 2 \int k^{\prime \prime \prime} \frac{\partial k^{\prime \prime \prime}}{\partial t}=2 \int k^{\prime \prime \prime}\left(k^{2} k^{\prime \prime}+k^{3}-\frac{L}{2 A} k^{2}\right)^{\prime \prime \prime} \\
= & -2 \int k^{(4)}\left(k^{2} k^{\prime \prime}+k^{3}-\frac{L}{2 A} k^{2}\right)^{\prime \prime} \\
= & -2 \int k^{(4)}\left[k^{2} k^{(4)}+2 k k^{\prime} k^{\prime \prime \prime}+2\left(k^{\prime}\right)^{2} k^{\prime \prime}+2 k\left(k^{\prime \prime}\right)^{2}\right. \\
& \left.+2 k k^{\prime} k^{\prime \prime \prime}+6 k\left(k^{\prime}\right)^{2}+3 k^{2} k^{\prime \prime}-\frac{L}{A}\left(k^{\prime}\right)^{2}-\frac{L}{A} k k^{\prime \prime}\right] \\
= & 2 \int\left[-k^{2}\left(k^{(4)}\right)^{2}-4 k k^{\prime} k^{\prime \prime \prime} k^{(4)}-2\left(k^{\prime}\right)^{2} k^{\prime \prime} k^{(4)}\right. \\
& -2 k\left(k^{\prime \prime}\right)^{2} k^{(4)}-3 k^{2} k^{\prime \prime} k^{(4)}-6 k\left(k^{\prime}\right)^{2} k^{(4)} \\
& \left.+\frac{L}{A} k k^{\prime \prime} k^{(4)}+\frac{L}{A}\left(k^{\prime}\right)^{2} k^{(4)}\right] .
\end{aligned}
$$

We use the same trick as in the proof of the previous lemma to bound the last seven terms by the first one and some additional penalty ones. 
Thus we get

$$
\begin{aligned}
\frac{d}{d t} \int\left(k^{\prime \prime \prime}\right)^{2} \leq & C_{1} \int\left(k^{\prime}\right)^{2}\left(k^{\prime \prime \prime}\right)^{2}+C_{2} \int \frac{\left(k^{\prime}\right)^{4}\left(k^{\prime \prime}\right)^{2}}{k^{2}}+C_{3} \int\left(k^{\prime \prime}\right)^{4} \\
& +C_{4} \int k^{2}\left(k^{\prime \prime}\right)^{2}+C_{5} \int\left(k^{\prime}\right)^{4}+C_{6} \int\left(k^{\prime \prime}\right)^{2}+C_{7} \int \frac{\left(k^{\prime}\right)^{4}}{k^{2}}
\end{aligned}
$$

where each term except the first is bounded by a constant (recall again that $k \geq k_{\min }(t) \geq k_{\min }(0) e^{-\left(L^{2}\right) /\left(16 A^{2}\right) t}$ has a positive lower bound). And the first term is bounded by a constant times $\int\left(k^{\prime \prime \prime}\right)^{2}$ which shows that the growth rate of $\int\left(k^{\prime \prime \prime}\right)^{2}$ is at worst exponential and that the quantity is finite on finite intervals.

Corollary 4.10. Under the same hypothesis as in the previous lemma, $k^{\prime \prime}$ is bounded.

Proof. In one-dimensional case

$$
\max |f|^{2} \leq C \int\left|f^{\prime}\right|^{2}+f^{2}
$$

and we apply this to $k^{\prime \prime}$.

Lemma 4.11. If $k, k^{\prime}$ and $k^{\prime \prime}$ are uniformly bounded, then so are $k^{\prime \prime \prime}$ and all the higher derivatives with respect to $\theta$.

Proof. We calculate:

$$
\begin{aligned}
\frac{\partial k^{\prime \prime \prime}}{\partial t}= & \left(k^{2} k^{\prime \prime}+k^{3}-\frac{L}{2 A} k^{2}\right)^{\prime \prime \prime} \\
= & k^{2} k^{(5)}+6 k k^{\prime} k^{(4)}+\left[6\left(k^{\prime}\right)^{2}+8 k k^{\prime \prime}+3 k^{2}-\frac{L}{A} k\right] k^{\prime \prime \prime} \\
& +\left[6 k^{\prime}\left(k^{\prime \prime}\right)^{2}+18 k k^{\prime} k^{\prime \prime}+6\left(k^{\prime}\right)^{3}-\frac{3 L}{A} k^{\prime} k^{\prime \prime}\right]
\end{aligned}
$$

If $k, k^{\prime}$ and $k^{\prime \prime}$ are bounded then the maximum principle can be applied to $k^{\prime \prime \prime} e^{\mu t}$ for suitably choosing $\mu$. On a finite time interval, this implies that $\left|k^{\prime \prime \prime}\right|$ is bounded.

Generally, if $k, k^{\prime}, \ldots, l^{(l-1)}$ are bounded, then

$$
\frac{\partial k^{(l)}}{\partial t} \leq k^{2} k^{(l+2)}+2 l k k^{\prime} k^{(l+1)}+C k^{(l)}+C
$$

shows that $k^{(l)}$ is bounded on finite intervals. 
Theorem 4.12. The solutions $k, L$ and $A$ to the initial value problem in Theorem 3.4 are continuous on the time interval $[0, \infty)$.

Proof. Theorem 3.4 has shown the short-time existence and uniqueness for the classical solutions. Now suppose that the solutions exist on the time interval $\left[0, T^{*}\right)$, with $T^{*}<\infty$, then from the above geometric, integral and pointwise estimates and Lemma 4.7, Corollary 4.10 and Lemma 4.11, it follows that $k$ and all its derivatives with respect to $\theta$ are bounded. Using the evolution equation (3.3) for curvature and inequality (2.21) we can bound the time derivatives of $k$ as well. Thus $k$ converges to a $C^{\infty}$ function at $T^{*}$ and defines a closed curve by using (3.6) in the previous section. Now, we use the short-time existence to extend the time interval on which the solution $k$ is defined. This shows that the solution $k(\theta, t)$ can be defined on the time interval $[0, \infty)$. Therefore the associated $L$ and $A$ can also be defined by using (3.7) and (3.9). Thus completes the proof.

\section{The proof of the main theorem}

Main theorem. A convex closed curve in the plane which evolves under (1.1)-(1.4) remains convex, expands the enclosed area while shortens its length during the evolution process and converges to a circle in the $C^{\infty}$ metric.

Proof. Since the tangential component of the evolution equation for the curve does not affect the geometric shape of the evolving curve, and therefore we can reduce the problem equivalently to (2.14)-(2.17). Now, Theorem 2.4 and Lemma 2.5 ensure that a convex curve evolving according to (2.14)(2.17) remains convex and makes the enclosed area increase and its length decrease.

As to existence, we have first shown in Theorem 3.3 that the evolution problem (2.14)-(2.17) is equivalent to an initial value problem of nonlinear differentio-integral equations (Theorem 3.3), and then shown that the later is both locally (Theorem 3.4) and globally solvable (Theorem 4.12).

To check convergence, one sees from Theorem 2.8 that the evolving curve converges to a circle in the Hausdorff metric, which can be considered as " $C$ " convergence. Now, mimicking the proofs of subsections 5.1-5.6 of [12], one can show that

$$
\frac{k_{\min }(t)}{k_{\max }(t)} \rightarrow 1
$$


as $t \rightarrow \infty$. This implies that the curvature $k$ converges to a constant $R$, which can be considered as " $C$ " " convergence to the circle. Finally, after a series of calculations and estimates similar to those of Section 5.7 of [12], one can prove by induction that for any $\alpha(0<\alpha<1)$ and any positive integer $l \geq 1$, there exists a constant $C(l)$ and sufficiently large time $T$ such that for $t>T$,

$$
\left\|k^{(l)}\right\|_{\infty} \leq C(l) e^{-(2 \alpha) /\left(R^{2}\right) t}
$$

This proves that all of the derivatives of $k$ converge to 0 as the time $t$ goes to infinity, which can be considered as the " $C$ " convergence to the circle.

\section{Acknowledgements}

We are grateful to the anonymous referee for careful reading of the original manuscript of this paper, giving us invaluable comments and pointing out several typos. The second author would like to thank Professors Guofu Lu and Chunli Shen and Dr. Min Dai for useful discussions relating to this paper. This work is supported in part by the National Science Foundation of China (No. 10671066) and Shanghai Leading Academic Discipline Project (No. B407).

\section{References}

[1] B. Andrews, Evolving convex curves, Calc. Var. Partial Differential Equations, 7 (1998), 315-371.

[2] B. Andrews, Non-convergence and instability in the asymptotic behavior of curves evolving by curvature, Comm. Anal. Geom. 10 (2002), 1409-1449.

[3] S. Angenent, On the formation of singularities in the curve shortening flow, J. Differential Geom. 33 (1991), 601-633.

[4] S. Angenent, G. Sapiro and A. Tannenbaum, On the affine heat equation for non-convex curves, J. Amer. Math. Soc. 11 (1998), 601-634.

[5] B. Chow, L.P. Liou and D.H. Tsai, Expanding of embedded curves with turning angle greater than - $\pi$, Invent. Math. 123 (1996), 415-429.

[6] B. Chow and D.H. Tsai, Geometric expanding of convex plane curves, J. Differential Geom. 44 (1996), 312-330. 
[7] K.S. Chou and X.P. Zhu, The curve-shortening problem, Chapman and Hall/CRC, Boca Raton, FL, 2001.

[8] W.J. Firey, Shapes of worn stones, Mathematika 21 (1974), 1-11.

[9] M.E. Gage, An isoperimetric inequality with applications to curve shortening, Duke Math. J. 50 (1983), 1225-1229.

[10] M.E. Gage, Curve shortening makes convex curves circular, Invent. Math. 76 (1984), 357-364.

[11] M.E. Gage, On an area-preserving evolution equation for plane curves, in Nonlinear problems in geometry, (ed., D.M. DeTurck), Contemporary Mathematics, Amer. Math. Soc., Providence, 51 (1986), $51-62$.

[12] M.E. Gage and R.S. Hamilton, The heat equation shrinking convex plane curves, J. Differential Geom. 23 (1986), 69-96.

[13] M. Grayson, The heat equation shrinks embedded plane curve to round points, J. Differential Geom. 26 (1987), 285-314.

[14] M. Grayson, Shortening embedded curves, Ann. of Math. 129 (1989), $71-111$.

[15] R. Hamilton, The formation of singularities in the Ricci flow, Surveys in Differential Geometry, Vol. II (Cambridge, MA, 1993), International Press, Cambridge, MA, 1995, 7-136.

[16] G. Huisken, Flow by mean curvature of convex surfaces into spheres, J. Differential Geom. 20 (1984), 237-266.

[17] G. Huisken, The volume preserving mean curvature flow, J. Reine Angew. Math. 382 (1987), 35-48.

[18] G. Huisken, Lectures on geometric evolution equations, Tsing Hua Lectures on Geometry and Analysis (Hsinhcu, 1990-1991), International Press, Cambridge, MA, 1997, 117-143.

[19] U.F. Mayer, A singular example for the averaged mean curvature flow, Experiment. Math. 10 (2001), 103-107.

[20] R. Osserman, Bonnesen-style isoperimetric inequalities, Amer. Math. Monthly 86 (1979), 1-29.

[21] S.L. Pan, A note on the general curve flows, J. Math. Study 33 (2000), $17-26$. 
[22] S.L. Pan, Local solvability of a system of nonlinear differentio-integral equations, Chinese J. Contemp. Math. 22 (2001), 167-176; Chinese Ann. of Math. Ser. A 22 (2001), 179-188, (Chinese).

[23] G. Sapiro and A. Tannenbaum, Invariant curve evolution and image processing, Indiana Univ. J. Math. 42 (1993), 985-1009.

[24] K. Tso, Deforming a hypersurface by its Gauss-Kronecker curvature, Comm. Pure Appl. Math. 38 (1985), 867-882.

[25] B. White, Evolution of curves and surfaces by mean curvature, Proc. ICM I (2002), 525-538.

Department of Applied Mathematics

TONGJI UNIVERSITY

Shanghai 200433

PeOple's Republic of ChinA

E-mail address: jiang_lishang@yahoo.com.cn

Department of Mathematics

EAst China Normal University

Shanghai 200062

People's Republic of China

E-mail address: slpan@math.ecnu.edu.cn

Received November 18, 2005 